\title{
Experiences and Perceived Effectiveness of Carbon-fibre Triplanar Orthotics for People Affected by Polio: A Qualitative Descriptive Study
}

Rachelle A. Martin DipPhys, MHealsc, PhD

Lecturer, Rehabilitation Teaching and Research Unit, Department of Medicine, University of Otago Wellington, Wellington, New Zealand; Research Lead, Burwood Academy of Independent Living, Christchurch, New Zealand

Anne C. Fitzpatrick BA(Hons), GradDipSc, MBA

Executive Leadership Coach and Consultant, Lead to Success, Wellington, New Zealand

William M. M. Levack BPhty, MHealSc, PhD

Professor, Rehabilitation Teaching and Research Unit, Department of Medicine and Dean and Head of Campus, University of Otago Wellington, Wellington, New Zealand

\section{ABSTRACT}

A new type of carbon-fibre triplanar orthotic (CTO) was recently introduced to New Zealanders affected by polio. This study aimed to assess CTO recipients' experiences and perceptions of these orthotics. A qualitative descriptive study based on semi-structured interviews was conducted to explore experiences of people who have had polio regarding the impact of CTOs on health and wellbeing, the process of training and adjustment, and how benefits from the CTOs were or were not achieved. Participants described substantial investments of time, energy and money needed to acquire and adapt to CTOs (Theme 1). They expected the CTOs to improve their posture, mobility, relieve pain and prevent deterioration in functioning. However, frequently there was a mismatch between reality and expectations (Theme 2). Ongoing orthotic and rehabilitation support plus sustained commitment and effort by CTO recipients contributed to benefits gained from these orthotics (Theme 3). When considering purchase of a CTO, people who have had polio should be aware of the time, energy, effort and personal cost required to fully benefit from the new orthotic. They should also be aware that individual responses to orthotics, including subjective reports of success, can be highly variable.

Martin, R. A., Fitzpatrick, A. C. \& Levack, W. M. M. (2021). Experiences and perceived effectiveness of carbon-fibre triplanar orthotics for people affected by polio: A qualitative descriptive study. New Zealand Journal of Physiotherapy, 49(3), 147-155. https://doi.org/10.15619/NZJP/49.3.05

Key Words: Orthotics, Poliomyelitis, Post-polio syndrome, Qualitative Research, Rehabilitation

\section{INTRODUCTION}

There is currently an estimated 9,750 people in New Zealand living with the effects of polio (Jones et al., 2016; Jones et al., 2017). Polio (also called poliomyelitis) is a highly infectious viral disease that devastated communities worldwide in the first half of the 1900s (Smallman-Raynor \& Cliff, 2006). New Zealand was no exception to this pandemic, with polio resulting in hundreds of deaths and long-term neuromuscular disabilities in the many thousands of people who survived the initial infection (Butterworth \& Ross, 1994; Ross, 1993; Wilson \& Baker, 2012). In addition, approximately $40 \%$ of those initially infected with polio went on to develop post-polio syndrome (Lin \& Lim, 2005). Post-polio syndrome is characterised by the onset of new or worsening muscle weakness 30-40 years after the original polio attack, resulting in new or exacerbated disabilities. Currently, many thousands of New Zealanders experience mobility restrictions, joint contracture, shortness of breath and problems with sleep, fatigue and pain due to both the original impairments sustained during polio in their youth and/or as a consequence of post-polio syndrome, or as a result of living with a long-term disability (Chetwynd et al., 1993; McNalley et al., 2015).
It is difficult to establish the exact number of polio survivors in New Zealand because there is no formal record for this. Many people who survived polio just returned to their prior lives after recovering from the initial infection, managing their muscle impairments, joint problems and breathing difficulties independently, and "rarely spoke again of those polio years" (Morris, 2013, p. 1). Polio survivors became increasingly forgotten by the New Zealand health system after the successful introduction of a vaccine in the 1950s (Stewart, 2015).

One of the key needs in New Zealand's polio community today is for specialist orthotic services (Jackman, 2016, 2017). People who have had polio may have idiosyncratic muscle impairments in their lower limbs, contributing to a variety of problems with mobility-related activities such as walking short and long distances, climbing stairs and performing various activities of daily living (Farbu, 2010). Musculoskeletal changes following polio and post-polio syndrome are different than those experienced by people with other types of neurological conditions, such as stroke or multiple sclerosis, so require different solutions to optimise physical functioning (Genêt et al., 2010; Portnoy \& Schwartz, 2013). 
The standard type of orthotic for people who have had polio in New Zealand is made of metal or solid plastic, can be ill-fitting and uncomfortable, and is limited in the assistance it provides during walking (Jackman, 2016). These types of orthotics are primarily designed to help prevent hyper-extension during the stance phase of gait and foot drop during the swing phase. However, they do not correct the many other problems with joint alignment experienced by people who have had polio - particularly issues of inversion and eversion at the ankle or internal rotation at the knee - and can be uncomfortable for some to wear. In 2018, in response to these challenges, people who have had polio in New Zealand began exploring options for accessing a new type of carbon-fibre triplanar orthotic (CTO) provided by orthotists outside of New Zealand. These new orthotics used carbon-fibre materials to provide lightweight, dynamic support in three dimensions of movement (flexion/ extension; inversion/eversion; internal/external rotation) and were promoted as having the potential to dramatically improve a person's functional abilities, reduce falls, reduce pain and discomfort, and reduce energy consumption during movement. The Duncan Foundation (a New Zealand registered charity for people living with neuromuscular conditions) began supporting individuals with polio to consider use of these new types of orthotics. They also began providing funding to support the physiotherapy required after people were fitted with a CTO However, these CTOs still required a significant financial investment for people who have had polio, since funding for the orthotics was not (and still is not) available through New Zealand's health system, nor could they be funded by private insurance companies. Therefore, there was a need to gather information on the effectiveness of these new orthotics to inform decisions about whether to invest in them.

There are several challenges with undertaking research in this area of clinical practice. First, the population of people who might benefit from these CTOs is relatively small, with a high degree of heterogeneity in their presenting impairments, making it difficult to standardise interventions for research purposes and making randomised controlled trial methods unsuitable. Second, because little research has previously been conducted in this area of clinical practice, there is very little information about what types of standardised outcomes measures would best detect improvements that might be meaningful to the people receiving the orthotics. Third, there is little information beyond anecdotal reports on optimal preparatory and followup physiotherapy that is required for people to benefit fully from these CTOs. As such, we conducted an initial qualitative study to explore the experiences and perceptions of people affected by polio and who had received CTOs, regarding: 1) the impact of CTOs on their health and wellbeing; 2 ) the process of adjustment when learning to live with CTOs, including reflections on both the formal (e.g., provided by health professionals) and informal (e.g., learning from experience) aspects of the adjustment process; and 3) the possible mechanisms of effect regarding how CTOs achieved or did not achieve positive health effects.

\section{METHODS}

\section{Research design}

We conducted a qualitative descriptive study, influenced by grounded theory methods (Charmaz, 2014), to gather data from people affected by polio who had been recently fitted with a CTO. Ethical approval for this study was granted by the University of Otago Human Ethics Committee (Health) (reference number H19/021). The research team comprised one person with lived experience of paralytic polio and post-polio syndrome in New Zealand ( $A F$, female, Pākehā) and two research physiotherapists with extensive experience of qualitative methods (WL, male, Pākehā and RM, female, Pākehā).

\section{Participant recruitment}

To be included in this study, participants needed to be adults diagnosed with polio, who had been fitted with a CTO within the past two years. The Duncan Foundation contacted recipients of the CTOs to elicit their willingness to participate in the study. At the time of the study (May 2019 to January 2020) only 10 people who have had polio in New Zealand had received a CTO. This study collected data from people who had already received their CTO and did not influence the way that CTOs were assessed or provided or how ongoing follow-up was delivered.

\section{Data collection}

Semi-structured interviews were conducted by one researcher (AF) via videoconference. The initial interview questions were developed by two researchers ( $\mathrm{AF}$ and $\mathrm{WL}$ ) based on issues raised in a prior survey on orthotic experiences of Polio NZ members (Jackman, 2016) and on the lived experience of one of the researchers (AF) (see Appendix A). We sent questions to the participants in advance so they could prepare for the interview. Interviews lasted between 60 and $90 \mathrm{~min}$, with one participant electing to provide written answers only. All interviews were digitally recorded and transcribed verbatim. We also collected demographic data including gender, age, ethnicity, age of onset of polio, limbs affected by polio, and orthotic and mobility aids used prior to the new CTO. We sought quantitative data on mobility before and after fitting of the orthotics (e.g., walking speed, endurance, balance) but this had not been routinely collected for all participants using standardised measurement methods so was not available for reporting.

\section{Data analysis}

We used NVivo 12 (QSR International) software to facilitate analysis and management of qualitative data. We used constant comparative methods to analyse the data (Charmaz, 2014). We read and reread each transcript, incorporating findings from additional data as the study progressed. We conducted initial data coding (open coding) on a line-by-line basis with subsequent analysis exploring relationships between codes to develop higher-order concepts. We did not have enough eligible participants to permit theoretical sampling or to test assumptions about reaching data saturation, so a full grounded theory study was not possible.

One researcher (AF) undertook initial analysis of all data, discussing and peer reviewing the data with a second researcher $(\mathrm{WL})$ as the study progressed. A third researcher (RM) independently read and coded all data to further enrich the range of interpretations of the dataset. The whole research team was involved in the development of the final analysis, which was reached by consensus. To further strengthen the credibility and trustworthiness of this analysis, we provided participants with 
a summary of findings and presented preliminary (anonymised) findings as a presentation at a national polio conference in New Zealand on 18 October 2019. Feedback during and following this conference supported the direction of the analysis and the conclusions we had reached.

\section{RESULTS}

\section{Participants}

Eight out of 10 people (six men, two women; age range 55 to 77 years) who had received CTOs agreed to be interviewed. One declined because he was unable to use the CTO for health reasons unrelated to the orthotic. The other person declining did not provide a reason. Five participants had single CTOs and three participants had bilateral CTOs. We interviewed the participants 2 to 20 months following their CTO being fitted. Seven participants used orthotics (callipers or standard-issue ankle-foot orthosis) prior to CTO provision. Three participants lived in a city, three lived in a provincial town and two participants lived rurally (Table 1).

\section{Table 1}

Participant Characteristics $(\mathrm{N}=8)$

\begin{tabular}{ll}
\hline Age & Mean: 67.9 (range 55-77) years \\
Gender & Male: $n=6$ \\
Ethnicity & Female: $n=2$ \\
& Mew Zealand European: $n=2$ \\
Type of CTO & Single ankle-foot orthosis: $n=4$ \\
& Bilateral ankle-foot orthoses: $n=2$ \\
& Single knee-ankle-foot orthosis: $n=1$ \\
Bilateral knee-ankle-foot orthoses: $n=1$ \\
Time since CTO & Mean: 13 (range 2-20) months \\
fitted & \\
\hline
\end{tabular}

Note. СTO = carbon-fibre triplanar orthotic.

\section{Overview of findings}

The impact of CTOs on participants' health and wellbeing varied greatly, with some participants describing the impact as being positively "life changing" (Participant [P]3), while others described the CTOs as being "ill advised, [a] waste of time and an expensive nonsense" (P2). For those participants who had experienced improvements in their functioning, the CTO allowed them to participate more fully in valued activities and life roles and had a positive impact on their wellbeing: "The period of time I can stand for in the brace is pretty much limitless ... it gives you a level of comfort and confidence that I never used to have" (P7).

However, of the eight participants, only one was using his CTO for most of his daily functioning, five were using their CTOs for part of their daily functioning and two were not using their CTOs at all. All participants described the process of adjustment as being both lengthy and energy intensive. At the time of the interview, none of the participants felt they had completed their journey to full use of their new orthotic. Some felt they had made no progress; others that they were about halfway to full adjustment to the orthotic; while most indicated that they were less than halfway. There were a variety of views as to whether
CTOs were worth the investment, with several saying it was too early to tell.

Three key themes have been identified from participants' experiences and perceptions of CTO use. The first theme (Substantial investments) relates to the investment of time, energy, money and support that was needed to acquire and adapt to the new orthotics. The second theme (Expectations and reality) examines the range of expectations that participants had of the difference CTOs would make to their lives and how closely the reality matched their expectations. The third theme (Contributors to success) explores factors that appeared to contribute to recipients perceiving that the CTO optimised their health and wellbeing and therefore that their investment had been worthwhile.

\section{Theme 1: Substantial investments}

Overall, participants talked about the significant investment of time, energy and money required to purchase and train to use the CTOs, with no guarantee of success. Financially, CTOs were a significant investment with the cost of CTOs varying between NZ\$10,000 to NZD $\$ 54,000$ per person (mean NZD $\$ 19,250)$. Participants funded their CTOs from a variety of sources, including loans from Polio NZ and other philanthropic organisations, via crowdfunding or at personal cost.

My total investment ... [is] in the vicinity of $\$ 20,000$ is expensive but I look at it from the point of view that if I want to retain my freedom, that's the price. That's what I am going to have to do. (P7)

Additional, usually unforeseen, costs were also incurred. These included costs associated with travel and accommodation for CTO assessments and follow-up appointments, treatment costs to attend physiotherapy sessions, vehicle modifications in response to altered posture or movement patterns, and purchase costs of parallel bars and other mobility equipment such as walking poles to allow the participant to practise using their CTOs. Participants often needed to purchase different footwear because the CTO would not fit in their existing shoes. Different size shoes were often required for each foot.

We had to buy shoes about five sizes too big because to get the width you have to go up in shoe size ... and it's compounded because you only need it on one leg and not on the other. (P8)

If the CTO was worn underneath participants' clothing, trousers also needed to be replaced to accommodate the CTO.

Physically preparing to get the CTOs and then learning to use them required a considerable investment of time and effort. Participants needed to undertake a daily stretching regime to improve their range of movement. They also needed to learn to stand and walk with new postures and movement patterns before they could use the CTOs functionally: "You've got to basically change your whole way of walking which you've been doing all your life, and you've got to change that, and it's a pretty difficult task" (P8).

Many began by putting their new orthotics on for just an hour every second day, increasing their duration and frequency of practice over time. Some participants ended up training several 
times each day: "When I got home, about four times a day I would get into my braces and just stand there between the parallel bars, initially just standing there and then starting to move my hips" (P3).

Donning and doffing the CTOs also took more time than previous orthotics, although participants reported that this became quicker with practice. Some participants reported that regular daily practice with the orthotic was not possible due to the time required to put on the CTO.

I should be spending more time but when I put them on I usually have them on for about an hour and I have tried to put them on the weekend because that's the only time when I've really got time to focus on them. (P1)

A particularly vexing issue for participants was the need to slowly adapt to using the CTO while also needing to continue to use their previous orthotics to participate in daily tasks. When they reverted to using their old orthotics, it was challenging to return to the movement patterns they used to function at previous levels.

The extensive practice and associated concentration required to learn to use the CTO also involved a considerable investment of mental and emotional energy. Many participants reported that the demands of adjusting to the new orthotics could lead them to feeling exhausted, discouraged and even despairing: "Coping with that feeling of despair is the thing I would say everyone is going through and I certainly had to deal with" (P3).

Theme 2: Expectations and reality

In this second theme, we explore the impact of CTOs on participants' ability to undertake a range of functions and how closely reality matched their expectations. Participants had a variety of expectations of the difference CTOs would make to their lives. Reasons given for wanting to change to CTOS included factors related to improved postural alignment (i.e., standing straighter, preventing further deformation), improved mobility (i.e., improved walking, reduced falling), improved functioning (i.e., looking after self, having the opportunity to do things not previously able to do), relieving pain (i.e., by reducing the load on specific joints or surfaces) and preventing deterioration in functioning (i.e., being able to stay out of a wheelchair, retaining ability to mobilise independently and keeping active in the community for longer). Other reasons for transitioning to CTOs included getting an orthotic that was stronger and less likely to break.

It was all about keeping me active and able to look after myself as I get older and also of course if I could walk better, you know that was the biggie. I mean you know if I could improve my walking and which of course I was limping more and more heavily as my legs deteriorated. (P6)

Postural alignment and standing

While not identified as being a key reason for wanting to transition to CTO use, improved postural alignment and endurance while standing emerged as one area in which participants noticed improvements. The CTOs appeared to contribute to the participants feeling they were able to stand with less effort for more extended periods, with improved postural alignment and with greater stability. This allowed participants to stand and converse with people more easily, which many participants reported was a significant benefit: "Before when I was talking with people I was looking around after about 5 minutes for someone to hang on to, but now I can just stand there for like half an hour and it's perfect" (P4).

Partners and other family members commented on how much straighter and/or taller the participant was standing. This ability to stand for more extended periods with less effort allowed participants to participate in activities and roles that were meaningful for them and had a positive impact on their wellbeing

\section{Mobility}

Improved walking was a fundamental expectation held by many participants. One participant (P4) reported that he could now walk double the distance that he had been able to before transitioning to the CTO. Another recipient described the positive difference in his walking and that moving had become "amazingly different and wonderful" (P3). However, many of the participants reported that while they had mastered walking on the flat or uphill without too much difficulty, they struggled with downhill, cambers and uneven ground.

It's fine going up a rise but going down a rise is dreadful. So, when I go to town, I've [still] got a crutch in the car and if I'm going to town to shop, I take the crutch with me. (P6)

Pain

Relieving pain was another key expectation that participants had of transitioning to using a CTO. Again, there were mixed responses as to whether CTO use impacted on participants' experience of pain. For some CTO wearers, their pain levels were reduced when wearing the CTO. For one participant (P3), the CTO had provided relief from the pain he had suffered for most of his life. Another (P1) commented that the pain in his knee and ankle joints had reduced because his ankles were held in fixed positions by the orthotics. However, for some participants, the changed movement patterns required to function while using the CTOs had contributed to the development of new pains in other joints. The participant who had reduced pain in his knees and ankle joints (P1) experienced new discomfort in his hips, for instance. Another reported having problems with his back as a result of wearing the CTO. He explained: "You can't limp with it; you have to walk properly and if I try and walk properly that's when it really hurts" (P2). As such, an increase in pain was an unanticipated reality for some participants, contrary to their expectations.

\section{Functional activities}

Another unanticipated reality for some participants was the negative impact that CTO use had on their ability to function within some of their daily activities. While one recipient found that performing activities such as "lifting, reaching up to heights, playing music, painting [were] just a million times better" (P3), many other participants found that the CTOs constrained their ability to undertake activities. While they were able to walk, they felt that the CTO was limiting their ability to undertake activities they had been previously able to do with their old orthotics, such as driving and gardening: "I quite 
quickly realised that even if I got walking properly on it, it was going to be a way big step down from what I had in terms of function" (P2).

Some mentioned finding it hard to bend down or kneel with the CTO on. Another observed that "as for twisting and turning and climbing ladders and walking on uneven ground, it was impossible" (P2). For other participants, substantial effort and time were needed to get to the point where the CTO did not constrain them. One participant (P6) reported that it had taken nine months of regular practice to get to the point where they could drive with their CTOs and walk around the supermarket without holding on to the trolley.

Theme 3: Contributors to success

Two key factors appeared critical to a successful transition to CTO use for participants: 1) access to ongoing orthotic support to adjust or modify the CTO and 2) access to rehabilitation support to facilitate and receive feedback on their movement retraining. The chances of success were also improved by sustained effort on the part of the recipient - to complete the required stretching exercises and to practise using the CTOs during mobilisation tasks and functional activities.

\section{Ongoing orthotic and rehabilitation support}

The importance of being able to access appropriate orthotic and rehabilitation support before and after the provision of the CTO was evident across all respondents, especially when this support was offered collaboratively and the clinicians worked together to solve problems.

[The local orthotist] has been with [CTO provider], he's been making them over here for [the CTO provider]; we've seen him a couple of times. [The physiotherapist] would bring [the local orthotist] across to assess me to see if she was doing [it] right you know. The whole thing's a big learning curve even for the hospital as well. (P4)

Equity of access was related to the physical location of the recipient in relation to services, access to orthotic expertise and access to a physiotherapist with neurological rehabilitation expertise on an ongoing basis.

For participants in this study, the CTOs were provided by a supplier who was based outside of New Zealand. Therefore, CTO recipients required support from a New Zealand-based orthotic provider for adjusting the CTOs in response to emerging issues related to fit, comfort, amount of movement and evolving postural changes over time. Publicly funded orthotic services in New Zealand varied in their willingness to assist CTO recipients. This meant there was considerable variation between participants in their ability to access this required ongoing support. One recipient felt that her foot was rolling within her new CTO and her local orthotic service identified a possible cause so could make adjustments. A small number of recipients were able to adjust their own orthotics. Some participants had follow-up video conversations with the US-based CTO provider and, in a few cases, videos of standing and walking were sent for gait retraining feedback.

However, participants felt that more support was required than could be provided via online videoconferencing with the CTO provider. Participants required a qualified person such as a physiotherapist with neurological rehabilitation expertise to train them both before and after the CTO had been provided. Those who did access rehabilitative support before the provision of the CTO frequently reported improvements in postural alignment and strength separate to that provided by the orthotic, with one participant speculating that "now I have strengthened the leg, maybe we could have done away with these dynamic braces" (P4).

Participants also felt that, after the CTO had been provided, regular reviews over time were needed for a successful transition to CTO use. As one participant observed: "there should be someone [who] assesses you every three months to make sure [you're] doing it right" (P1). A small number of recipients were able to access physiotherapy services in their local publicly funded health services. The Duncan Foundation provided limited physiotherapy support to participants who were able to travel to the physiotherapist before and after fitting of the CTO. Others, however, did not have this opportunity and either had to pay for private physiotherapy or manage without any rehabilitative support: "They sort of gave you some suggestions and gave you a bit of assistance ... it's very much relying on the individual to actually do the work" (P8).

Participants who did not receive this support were often unsure whether their gait technique was correct and were fearful that they would have to start over again if they made mistakes in their retraining: "What bugs me the most is that there is so much uncertainty. I don't know if I am doing things right" (P5).

One participant (P3) reported having to start again with learning correct movement patterns when the CTO provider gave him feedback that his new walking technique was suboptimal.

Sustained commitment and effort

All participants stressed the need for sustained attention to motor relearning over an extended period.

The first six months of it is just all about retraining your mind, retraining how you stand, retraining the basic movements and once you've got those down, then I think the development after that comes a lot quicker. (P8)

Participants described that a great deal of self-discipline, perseverance and tenacity was required, stating that "you've really just got to drive yourself to stick with it and do the exercises" (P6). Participants talked about the importance of being "of a mindset to put in that work" (P6) to ensure a successful transition to CTO use. One participant noted that he had underestimated the need to do preparation exercises: "If you do a lot of work training yourself to stand properly before you get this thing, it would be a lot more effective and a lot easier, I think, to adapt to it" (P8).

For some participants, other daily responsibilities and activities made this sustained commitment difficult and, in some cases, unachievable. Some were unable to do the training needed because of their other health conditions. Participants who were in full-time paid employment reported that it was challenging to find the time and energy to practise wearing the new CTO, with one participant (P6) taking a month off work to concentrate on 
adjusting to the orthotic and another stating that "I put them on [at] the weekend because it is the only time when I really have time to focus on them" (P1).

\section{DISCUSSION}

The accounts of participants in this study demonstrate the variability in outcomes that can occur when people who have had polio receive new CTOs. Participants were more likely to find the experience of receiving a CTO worthwhile when they were able to access ongoing orthotic support to adjust or modify the CTO, when they received rehabilitation support to facilitate gait retraining and when they had the time and resources required to access and then learn to use their new orthotics. The chances of success were also improved by sustained effort on the part of CTO recipients.

One prior qualitative study of orthotic use by people with neuromuscular conditions (including some people with experiences of polio) has also highlighted the variability in outcome experiences from new orthotics - ranging from very positive to very negative (McCaughan et al., 2019). This prior study identified that any transition from an old to new orthotic could be challenging, making people reluctant to move to a new orthotic. The new information that our study adds is the extent of structured therapeutic exercise that might be required before any new orthotic is useable - particularly when the new orthotic requires people to relearn movement patterns. For some participants in our study, this additional therapeutic exercise amounted to several hours of self-directed training each week over a period of many months.

These findings highlight the importance of comprehensive assessment and establishing clear expectations when prescribing costly interventions such as CTOs. Assessments before fitting of an orthotic need to focus on more than just the biomechanical aspect of movement but also should consider when and how people will be able to complete all of the movement retraining required to fully benefit from their new orthotics - within the context of their individual supports, resources and daily lives. Exploring people's goals, priorities and anticipated outcomes, while also providing information about potential challenges, is likely to facilitate a more successful transition to CTO use. Potential recipients may benefit from talking to peers who have already had a CTO fitted, as discussion with experienced peers is known to aid informed decision making (Hammell, 2007; O’Neill et al., 2007).

The experiences of participants in this study also indicated how challenging it is for people with long-term health conditions to find and access the health services they need over their lifespan. Some participants in this study reported that the intensive physical training they completed resolved some of the problems with pain and functioning they had been experiencing before even being fitted with the CTO. However, the participants in this study reported that this type of rehabilitation support to meet their ongoing and changing health needs was often not easy for them to access in their usual healthcare services.

We initially started this study as a result of a call from the polio community in New Zealand for more research to be conducted on the cost-effectiveness of orthotics. This study however illustrates several reasons why establishing a strong evidence base in this area is difficult. The population of people who might benefit from CTOs is relatively small for research purposes. Recruitment in clinical trials is notoriously difficult. For instance, almost half of all publicly funded clinical trials in the UK fail to meet their target sample size due to problems with participant recruitment (Walters et al., 2017). Recruitment rates are not just based on the number of people with a condition in a population but also on the eligibility of people to participate when considering exclusion criteria, the willingness of people to enrol in a randomised controlled trial - considering for instance that they may not receive the treatment they are hoping for - the ability of people to get to a clinical centre where a study is being conducted, the timing of the trial for when a person is ready for a particular intervention and so forth. Participant recruitment also must account for dropout rates. For comparison, only one third of stroke survivors who are screened to join clinical trials of stroke rehabilitation are actually recruited, with a median dropout rate of $6 \%$ (interquartile range $13 \%$ ) of the people randomised to treatment groups (McGill et al., 2020). People who have had polio in New Zealand are highly diverse from a clinical perspective, relatively few in number in comparison to other conditions and geographically dispersed, making fully powered clinical trials very difficult to conduct.

As suggested by our study there is a high degree of variability in presentation, both biomedically (e.g., the nature and range of impairments) and psychosocially (e.g., social support, economic wealth, competing priorities, personal goals, attitude and resilience), which impact on an individual's response to CTOs. The orthotic itself is highly unlikely to influence changes in health and wellbeing without extensive additional therapy. The comprehensive additional therapy could itself have a large effect on the health and wellbeing of people who have had polio so would also need to be studied separately. This makes it difficult for the polio community to conduct the kinds of studies that are typically required for a strong economic argument to support new health funding from the public health sector or insurance providers. There is, therefore, a need to embrace 'practice-based evidence pathways' rather than solely 'evidence-based practice pathways' to gather meaningful evidence to support personal decision making and public health policy in this area of clinical practice, as recently advocated by Ogilvie et al. (2020).

Alternative research designs are needed to provide quantitative evidence supporting clinical and policy decision making around the funding of orthotics for people who have had polio.

For example, single-case experimental designs (SCEDs) can rigorously demonstrate whether a treatment effect is evident for individual participants, while also exploring the range of responses to interventions (Kratochwill et al., 2013). Synthesised findings from a number of SCEDs can support the development of evidence-based practice by contributing knowledge not only about whether an intervention works in controlled and ideal circumstances but also on how it may work, for the range of people who access it (Shadish et al., 2014). This study suggests that future SCEDs on the topic of new orthotics for people with experience of polio could usefully focus on outcomes related to individually meaningful occupational performance, duration of time in standing per day and health-related quality of life. 
Although we invited all potential participants in New Zealand to participate in this study (with only two declining), this study was limited by its small sample size, which reduced the range of experiences and opinions that we could draw on to develop these results. Furthermore, we did not have access to quantitative clinical data on the participants' impairments and functioning before and after receiving their orthotics, making it more difficult to consider the transferability of these findings to other people in other settings.

When considering the data from this study, it is important to be aware of the context in which they were collected. The participants in this study were the first people in New Zealand with polio to receive CTOs. The knowledge gained from these first cases and this study is likely to change the experiences of any people who have had polio who might decide to be fitted with CTOs in the future as they will be better prepared for the experience. Likewise, the experience of people receiving CTOs in other countries may differ depending on the supports and services that they have available to them. These findings will help guide the development of protocols and selection of outcome measures in future research on orthotics for people who have had polio.

\section{CONCLUSION}

When considering the purchase of a СTO, people who have had polio need to be aware of the time, energy, effort and personal cost that may be required to fully benefit from the new orthotic. They should also be aware that individual responses to orthotics, including subjective reports of success, can be highly variable. Research questions about the cost-effectiveness of these types of orthotics for people who have had polio are not suitable for randomised controlled trial designs, and other types of research approach, such as SCEDs, may be more useful to guide public policy and personal choice in this area of healthcare.

\section{KEY POINTS}

1. There is a high degree of variability among people affected by polio in terms of clinical presentation and responses to new orthotics.

2. When deciding to be fitted with a new, complex orthotic, people affected by polio need to have a thorough understanding of the commitment required in terms of money, time and effort to benefit from that orthotic fully.

3. Learning to use and fully benefit from a new carbon-fibre triplanar orthotic can take years, with ongoing input from orthotists and physiotherapists, so should be planned for when a new orthotic is being considered and fitted.

\section{DISCLOSURES}

This study was funded by a Lottery Health Research Grant from the Lottery Grant Board, New Zealand. There are no conflicts of interest that may be perceived to interfere with or bias this study.

\section{PERMISSIONS}

Ethical approval was obtained from the University of Otago Human Ethics Committee (Health) (reference number H19/021). All participants provided informed consent.

\section{ACKNOWLEDGEMENTS}

We would like to thank all the participants who gave their time for the study and the Duncan Foundation and Polio NZ who supported this research. In particular, we thank Gordon Jackman for his endless enthusiasm and support.

\section{ADDRESS FOR CORRESPONDENCE:}

Professor William Levack, Rehabilitation Teaching and Research Unit, University of Otago Wellington, Wellington, New Zealand.

Email: william.levack@otago.ac.nz

\section{REFERENCES}

Butterworth, K. P., \& Ross, J. C. (1994). Mind over muscle: Surviving polio in New Zealand. Dunmore Press

Charmaz, K. (2014). Constructing grounded theory (2nd ed.). Sage Publications.

Chetwynd, J., Botting, C., \& Hogan, D. (1993). Postpolio syndrome in New Zealand: A survey of 700 polio survivors. New Zealand Medical Journal, 106(964), 406-408.

Farbu, E. (2010). Update on current and emerging treatment options for post-polio syndrome. Therapeutics and Clinical Risk Management, 6, 307-313. https://doi.org/10.2147/tcrm.s4440

Genêt, F., Schnitzler, A., Mathieu, S., Autret, K., Théfenne, L., Dizien, O., \& Maldjian, A. (2010). Orthotic devices and gait in polio patients. Annals of Physical and Rehabilitation Medicine, 53(1), 51-59. https://doi. org/10.1016/j.rehab.2009.11.005

Hammell, K. W. (2007). Experience of rehabilitation following spinal cord injury: A meta-synthesis of qualitative findings. Spinal Cord, 45(4), 260 274.

Jackman, G. (2017, November 6). Polio survivors in 21st century New Zealand - "We're still here". Corpus. https://corpus.nz/polio-survivors-21st-centurynew-zealand-still/

Jackman, G. (2016). Orthotics users' survey of members 2016. Polio NZ Inc. https://www.researchgate.net/publication/304567603_Polio_NZ_Inc_ Orthotics_Users'_Survey_of_Members_2016/download

Jones, K. M., Balalla, S., Theadom, A., Jackman, G., \& Feigin, V. L. (2016). Prevalence of polio: An international systematic review (2016) [unpublished manuscript]. Duncan Foundation.

Jones, K. M., Balalla, S., Theadom, A., Jackman, G., \& Feigin, V. L. (2017). A systematic review of the worldwide prevalence of survivors of poliomyelitis reported in 31 studies. BMJ Open, 7, e015470. https://doi.org/10.1136/ bmjopen-2016-015470

Kratochwill, T. R., Hitchcock, J. H., Horner, R. H., Levin, J. R., Odom, S. L., Rindskopf, D. M., \& Shadish, W. R. (2013). Single-case intervention research design standards. Remedial and Special Education, 34(1), 26-38. https://doi.org/10.1177/0741932512452794

Lin, K. H., \& Lim, Y. W. (2005). Post-poliomyelitis syndrome: Case report and review of the literature. Annals of the Academy of Medicine, Singapore, 34(7), 447-449.

McCaughan, D., Booth, A., Jackson, C., Lalor, S., Ramdharry, G., Connor, R. J., Phillips, M., Bowers, R., \& McDaid, C. (2019). Orthotic management of instability of the knee related to neuromuscular and central nervous system disorders: Qualitative interview study of patient perspectives. BMJ Open, 9, e029313. https://doi.org/10.1136/bmjopen-2019-029313

McGill, K., Sackley, C. M., Godwin, J., McGarry, J., \& Brady, M. C. (2020). A systematic review of the efficiency of recruitment to stroke rehabilitation randomised controlled trials. Trials, 21, 68. https://doi.org/10.1186/ s13063-019-3991-2

McNalley, T. E., Yorkston, K. M., Jensen, M. P., Truitt, A. R., Schomer, K. G., Baylor, C., \& Molton, I. R. (2015). Review of secondary health conditions in postpolio syndrome: Prevalence and effects of aging. American Journal of Physical Medicine \& Rehabilitation, 94(2), 139-145. https://doi. org/10.1097/PHM.0000000000000166 
Morris, E. (2013). Carer stories: Polio then and now. Carers NZ. http://carers. net.nz/information/carer-stories-polio-then-now/

O'Neill, T., Jinks, C., \& Ong, B. N. (2007). Decision-making regarding total knee replacement surgery: A qualitative meta-synthesis. BMC Health Services Research, 7, 52. https://doi.org/10.1186/1472-6963-7-52

Ogilvie, D., Adams, J., Bauman, A., Gregg, E. W., Panter, J., Siegel, K. R., Wareham, N. J., \& White, M. (2020). Using natural experimental studies to guide public health action: Turning the evidence-based medicine paradigm on its head. Journal of Epidemiology and Community Health, 74, 203208. https://doi.org/10.1136/jech-2019-213085

Portnoy, S., \& Schwartz, I. (2013). Gait characteristics of post-poliomyelitis patients: Standardization of quantitative data reporting. Annals of Physical and Rehabilitation Medicine, 56(7-8), 527-541. https://doi.org/10.1016/j. rehab.2013.06.005

Ross, J. C. (1993). A history of poliomyelitis in New Zealand [Unpublished master's thesis]. University of Canterbury.

Shadish, W. R., Hedges, L. V., \& Pustejovsky, J. E. (2014). Analysis and metaanalysis of single-case designs with a standardized mean difference statistic: A primer and applications. Journal of School Psychology, 52(2), 123-147. https://doi.org/10.1016/j.jsp.2013.11.005
Smallman-Raynor, M. R., \& Cliff, A. D. (2006). Poliomyelitis. A world geography: Emergence to eradication. Oxford University Press.

Stewart, A. (2015, October 16). Polio victims: 'Society's forgotten about us'. Stuff. https://www.stuff.co.nz/national/72967707/polio-victims-societysforgotten-about-us? rm=m

Walters, S. J., Bonacho dos Anjos Henriques-Cadby, I., Bortolami, O., Flight, L., Hind, D., Jacques, R. M., Knox, C., Nadin, B., Rothwell, J., Surtees, M., \& Julious, S. A. (2017). Recruitment and retention of participants in randomised controlled trials: A review of trials funded and published by the United Kingdom Health Technology Assessment Programme. BMJ Open, 7, e015276. https://doi.org/10.1136/bmjopen-2016-015276

Wilson, N., \& Baker, M. G. (2012). Celebrating 50 years of polio elimination in New Zealand: But inadequate progress in eliminating other vaccinepreventable diseases. New Zealand Medical Journal, 125(1365), 67-74 


\section{Appendix A}

\section{INTERVIEW QUESTIONS}

1. Could you please briefly tell me about your experiences of polio to date?

Prompts

When did you get polio; where were you; what happened?

What parts of your body were affected and in what way?

How did having polio influence your life?

2. Could you please tell me about your past experience of orthotic services?

Prompts

What's it been like?

What kind of orthotics have you worn? For how long?

How did that make you feel? What do you think about that?

What operations, if any, have you had to assist you with your mobility?

What other health services have you used to assist you with your mobility?

What relationship did you have with your orthotist?

3. What has been your experience of getting dynamic braces?

Prompts

What led you to decide to get dynamic braces?

What did you expect it might be like to get dynamic braces?

What were your expectations for the braces?

What problems were you having that you hoped the dynamic braces would address?

What was it like to be assessed for the dynamic braces?

What was it like to be cast for the dynamic braces?

What was it like to be fitted for the dynamic braces?

What preparation did you do prior to receiving the braces? Any exercises?

4. What has been your experience of adjusting to the braces?

Prompts

What have you had to do to adjust to wearing the braces?

What have you had to do to learn to use the braces effectively?
What has that involved?

What support have you had in learning to adjust to the braces?

Do you have any comments on the cost of the dynamic braces and funding them?

What resources were you supplied to guide your training?

How well did you understand the information you were given?

How well do you understand gait functions?

What problems have you experienced since fitting (e.g. range of motion, muscular control, pain, body image etc.)?

5. What has been the impact on you of having the braces on your health and wellbeing?

Prompts

Physically

Socially

Emotionally

Psychologically

Financially

Pain levels

Fatigue levels

Clothing and footwear

How the orthotic looks and how it affects how you look How you function day to day

Your work

On your family

6. Overall, how worthwhile have the orthotics been for you?

Other prompts

Consider mechanism of effect:

How did the braces lead to that effect?

What other factors were involved in leading to that effect?

Why or how did that happen?

What did you do in order to gain/alleviate this effect? 